\title{
Comparative Study of Gait Gender Identification using Gait Energy Image (GEI) and Gait Information Image (GII)
}

\author{
Rosa Andrie Asmara ${ }^{1 *}$, Irtafa Masruri ${ }^{1}$, Cahya Rahmad $^{1}$, Indrazno Siradjuddin ${ }^{1}$, Erfan Rohadi ${ }^{1}$, Ferdian Ronilaya ${ }^{1}$, Anik \\ Nur Handayani ${ }^{2}$ and Qonitatul Hasanah ${ }^{1}$ \\ ${ }^{1}$ State Polytechnic of Malang \\ ${ }^{2}$ State University of Malang
}

\begin{abstract}
Identifying gender from the pedestrian video is one crucial key to study demographics in such areas. With current video surveillance technology, identifying gender from a distance is possible. This research proposed the utilization of computer vision to identify gender based on their walking gait. The data feature used to determine gender based on their walking gait divided into five parts, namely the head, chest, back, waist \& buttocks, and legs. Two different methods are used to perform the real-time gender gait recognition process, i.e., Gait Energy Image (GEI) and Gait Information Image (GII), while the Support Vector Machine (SVM) method used as the data classifier. The experimental results show that the process of identifying gender based on walking with GEI method is $55 \%$ accuracy and GII method is $60 \%$ accuracy. From these results, it can conclude that the method GII with SVM classifier has the best accuracy in the process of gender classification
\end{abstract}

\section{Introduction}

Research about gender recognition based on gait is now an exciting and challenging study. If the computer can recognize gender, it is indeed beneficial in various aspects of life. For example, a gender classification can identify how many customers visit a store, fashion analysis, make robots possible to determine gender, etc. GII method and GEI method are widely used in the process of gender classification based on gait. Therefore, we try to compare the two methods, which produce the best accuracy for real-time gender recognition based on gait.

Gender classification can do on fingerprint, face, iris and voice recognition, but when using the approach requires a coherent subject, proximity tools used for the process gender recognition and physical contact. This method cannot use if you want to recognize the object remotely, therefore, in this research, we do gender recognition based on gait. Gait is the process of identifying individuals from how they walk. The gait approach is somewhat incomplete as clothing styles and environmental factors also influence the process of gender recognition. What's more, when something happens to a person's physical condition like an injured foot, it can change the person's walking style. Despite the significant differences that make this approach not unique as fingerprint or iris, but the inherent gait characteristic of an individual remains, and it is irreplaceable, and it is useful in visual surveillance. The characteristic of a typical person's walking style makes it invaluable in the process of gender recognition.

In recent years, research on gender recognition has done. Han et al. proposed marking the character of human walking for individual recognition by gait with a new spatiotemporal approach to the representation of forces, called Gait Energy Image (GEI). Based on the research, it found that individual recognition by gait with GEI approach is effective and efficient and has a competitive performance [1]. Research on individual recognition by GEI method has also done by Chang et al. [2]. In the study using GEI method as a discriminatory characteristic through different angles and approaches. Based on experiments, shows that the system has achieved excellent performance in real-time and can apply to real-world applications. Yu et al. [3] classified genders based on human gait by combining psychological approaches using automated methods to improve the quality of accuracy. In the study mentioned that head and hair, back and chest are the most dominant part of the process gender recognition than other body parts. In a controlled environment, the gait classification is feasible despite many obstacles in the application process. The obstruction caused by the many variations of human appearance, change of clothes and shoes or when carrying objects.

\footnotetext{
* ${ }^{*}$ orresponding author: rosa.andrie@polinema.ac.id
} 
Chunli et al. [4] propose 2G (2D) 2PCA) Enhanced Gait Energy Image (EGEI) and 2-Directional 2dimensional principles component analysis ((2D)2PCA) for classification of human behaviour. The experimental results show that this algorithm is simple, achieving a higher classification accuracy with a short time. Mikawa et al. [5] have conducted research that is making a system can provide book recommendations to library visitors based on gender and visitor age in real time using silhouette-based gait classification. The classification process uses the Super Vector Machine (SVM) method which produces an accuracy of $77.5 \%$. Juang et al. [6] present the initial design and experimental results of gender recognition from walking movements using the GEI method with denoised energy image (DEI) in the pre-processing process. Support Vector Machine (SVM) used for training process and feature extraction. The results show the proposed method will adopt some typical values, but the accuracy can reach $100 \%$.

Zhang et al. [7] proposed an approach to integrate information from the multi-view gait at the feature level. The proposed method is the fusion scheme, showing more effective performance for multi-view gait-based gender classification. Arora et al. [8] also conduct research to recognize humans through gait. This paper presents the features of gait image based on the theory sets of information, then called image feature information gait. This paper presents gait image features based on the information set theory; henceforth these are called gait information image features. The proposed Gait Information Image (GII) derived by applying the concept of the information set on the frames in one gait cycle and two elements named Gait Information Image with Energy Feature (GII-EF) and Gait information image with Sigmoid feature (GII$\mathrm{SF})$ extracted. The classification process uses Nearest Neighbour (NN) to identify gait. Guru et al. [9] conducted research on gender recognition use robust feature level fusion of four directional vector's (i.e. horizontal, vertical, forward and backward diagonal). For each image sequence, GEI (Gait Energy Image) firstly constructed. Next, GGEI (Gradient Gait Energy Image) is obtained based on neighbourhood gradient computation. Then, variations in all four directions used as discriminative gait features. Subsequently, classification process uses Support Vector Machine (SVM), and testing process uses considerably largest multi-view CASIA - B (Chinese Academy of Sciences) dataset. According to the researchers, the results obtained include promising.

Based on the literature study, currently, GII method and GEI method are widely used in the process of gender classification based on gait. Therefore, the purpose of this study is gender classification in realtime based on gait by comparing GEI and GII methods. The best accuracy of both methods can utilize for further research.

\section{Material and Method}

\subsection{Pre-Processing Stage}

In this section, we will explain the pre-processing image before do the feature extraction. Pre-processing flowchart process is shown in Fig. 1. This research is a development of previous research, namely gender identification based on gait, which distinguishes from previous research is the gender identification in real-time with two method that is GII and GEI.

The process of making images into silhouettes using Otsu thresholding method. In the flowchart, there is a process cropping data according to the widest silhouette form. We chose the widest silhouette shape to make it easier to unify the image. When selecting a non-wide image then the unifying process creates an image that is not precise.

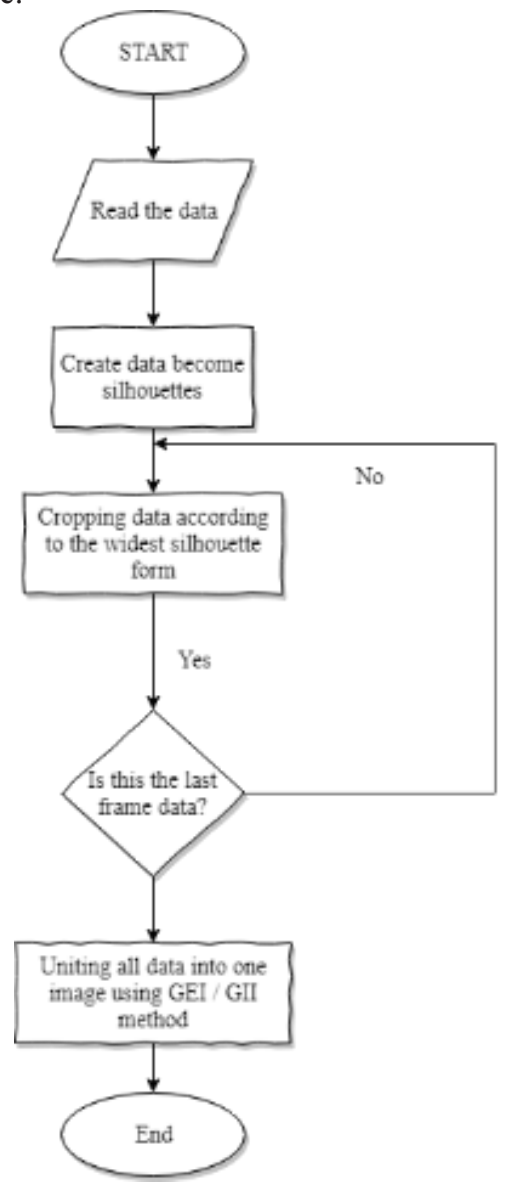

Fig. 1. Pre-processing flowchart on the image before feature extraction.

\subsection{Feature Extraction}

Before the gender recognition process automatically, we first collect the dataset first. Total 20 people $(9$ men, 
11 women) participated in this experiment. Based on earlier studies [3], in the human observed process shows that the upper body accounted for more information from the lower body in the classification gender.

In our experiments, all videos were captured a by fixed cameras, Logitech c525. Human silhouettes can be extracted using background subtraction and threshold.

When we use all the silhouette in the gait cycle as a feature, the dimension feature will be very high. The Gait Energy Image (GEI) can significantly reduce the progress dimension of the feature. GEI, also known as the average silhouette, a kind of Statistical features.

GEI has been said to be a good feature in gait recognition because of strong silhouettes errors and noise. Define GEI as

$$
F(i, j)=\sum_{t=1}^{T} I(i, j, t)
$$

Where $T$ is the number of frames in the sequence $I(i, j,),. I(i, j, t)$ is a binary silhouette image at frame $t, i$, and $j$ are the image coordinates. The silhouettes in a gait cycle and the GEI are shown in Fig. 2.
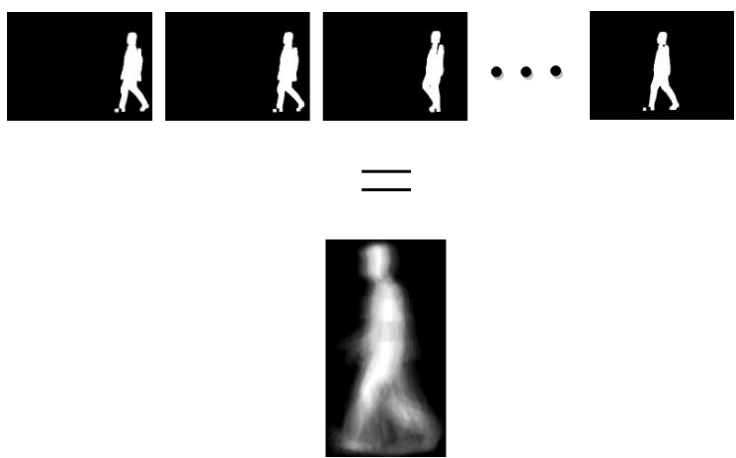

Fig. 2. Gait energy image (GEI) is the average silhouette in the gait cycle.

In addition to using the GI method, in our feature extraction process using GII. GII calculated for each pixel of the image through a complete cycle. Fuzzy sets can form the silhouette of the intensity values at particular pixel locations $X k_{i j}$ across all $\mathrm{K}$ frames of the gait cycle and the appropriate membership function.

\subsection{Design system}

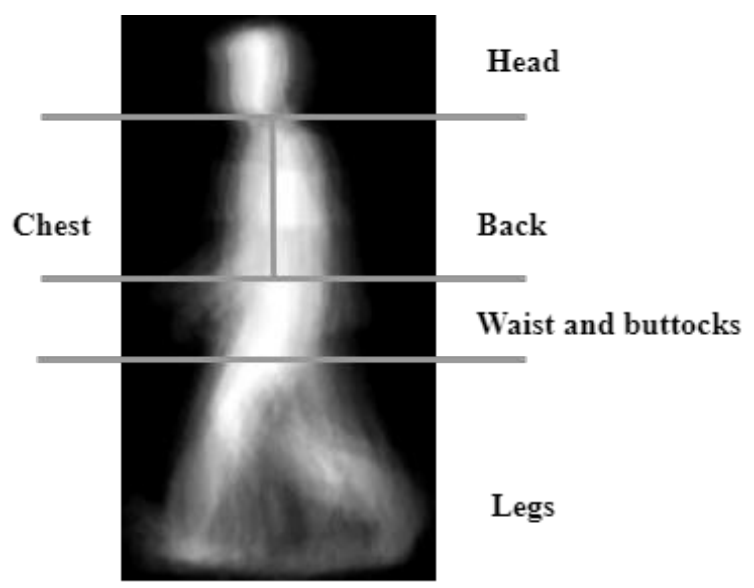

Fig. 3. Divide body into five parts using GEI and GII to recognize gender

Gender recognition process is executed in real-time using C\# programming language .NET software. In this study, feature data used to determine the gender based on walking gait divided into five parts, namely the head, chest, back, waist and buttocks also legs are shown in Fig. 3.

The design of real-time gender recognition system shown in Fig. 4. the process will be described as follows:

- The camera captures an ongoing human image.

- The pre-processing process completed making an image as the silhouette by using Otsu threshold method.

- The result of pre-processing, calculated using GII and GEI methods to generate the image already shown in the process diagram.

- Gender classification process conducted so the system can know the gender human being by walking gait. 


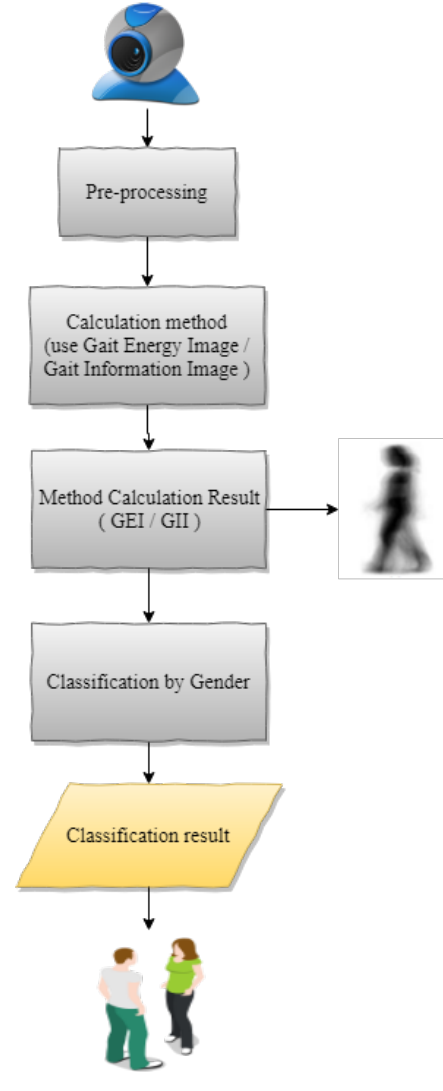

Fig. 4. Diagram process of the real-time system to gender classification by gait.

\subsection{Classification process}

In this research we use Support Vector Machine (SVM) method, the system classifies to produce two classes, that is class of men and class of woman. Support Vector Machine (SVM) is proven to produce an effective system in the process of gender classification $[5,6,9]$.

Support vector machine (SVM) is a technique to make predictions, both in case of classification and regression. SVM is in a class with the Artificial Neural Network (ANN) in terms of function and problem conditions that can be solved. Both are in the supervised learning class.

\section{Results}

The dataset collection process used 20 participants consist of 11 women and 9 men. The next process, making the image into silhouette using Otsu threshold method. Selected widest silhouette to aid in unifying the image. After that, the image preprocessing stage is calculate during GII and GEI methods followed by the classification process using the Support Vector Machine (SVM) method.

The classification process of gender classification gets the value which attached in Table 1. Based on Table 1. The classification process use cross-validation with $10 \%$ number of vold.
Table 1. The result of classification with SVM method.

\begin{tabular}{|c|c|c|c|c|}
\hline Method & Classification & Accuracy & Precision & Recall \\
\hline GII & SVM & $70 \%$ & $72.70 \%$ & $72.70 \%$ \\
\hline GEI & SVM & $60 \%$ & $63.60 \%$ & $63.60 \%$ \\
\hline
\end{tabular}

Refer to Table 1, discerning that in SVM classification, using GII method values points better than GEI. The accuracy of GII method that is $70 \%$ with recall and precision $72.7 \%$, while accuracy using GEI method that is $60 \%$ with recall and precision $63.6 \%$. The best results in terms of accuracy, precision, and recall obtained by the GII method.

\section{Conclusions}

In this research, the accuracy of our computer systems observed and analyzed. In the process of classification method using Support Vector Machine (SVM) with crossvalidation $10 \%$ number of vold, achieving $70 \%$ classification accuracy and $72.7 \%$ recall \& precision in GII method. Using the same classification method, in GEI method that is $60 \%$ with recall and precision $63.6 \%$. It can conclude that using GII method (Gait Information Image) has the highest accuracy in gender recognition process.

For additional research, we will try to increase the dataset and try to explore other features in this study so the results obtained can be much better.

\section{References}

1. H. Han, J. Ju, Bir Bhanu, Gait energy image representation: comparative performance evaluation on USF HumanID database, Proc. Joint Int'l Workshop VS-PETS 133-140 (2003)

2. C. Chang, Ping-Chieh, Ming-Chun Tien, Ja-Ling Wu, Chuan-Shen Hu, Real-time gender classification from human gait for arbitrary view angles, Multimedia, 2009. ISM'09. 11th IEEE International Symposium on 88-95 (2009)

3. Y. Yu, S. Shiqi, Tieniu Tan, Kaiqi Huang, Kui Jia, Xinyu Wu, A study on gait-based gender classification, IEEE Transactions on image processing 18, 8, 1905-1910 (2009)

4. C. Lin, Wang Kejun, A behavior classification based on enhanced gait energy image, Networking and Digital Society (ICNDS), 2010 2nd International Conference on 2 589-592 (2010)

5. M. Mikawa, M. Masahiko, Soichi Izumi, Kazuyo Tanaka, Book recommendation signage system using silhouette-based gait classification, Machine Learning and Applications and Workshops (ICMLA) 2011 10th International Conference on 1 416-419 (2011)

6. J Juang, Li-Hong, Shin-An Lin, Ming-Ni Wu, Gender recognition studying by gait energy image classification, Computer, Consumer and Control (IS3C), 2012 International Symposium on 837-840 IEEE (2012) 
7. Z De, Yahui Wang, Using multiple views for gaitbased gender classification, Control and Decision Conference (2014 CCDC), The 26th Chinese 21942197 IEEE (2014)

8. A. Parul, M. Hanmandlu, Smriti Srivastava, Gait based authentication using gait information image features, Pattern Recognition Letters 68 336-342 (2015)

9. G Guru, V. G. Manjunatha, V. N. Kamalesh, R. Dinesh, Human gait recognition using four directional variations of gradient gait energy image, Computing, Communication and Automation (ICCCA), 2016 International Conference on, 1367-1371 IEEE (2016)

10. M Hanmandlu, M Madasu, AnirbanDas, Contentbased image retrieval by information theoretic measure, Defence Science Journal 61, 5, 415-430 (2011). 